Documentation et bibliothèques

DOCUMENTATION BIBLIOTHEQUES

\title{
L'automatisation de la Bibliographie nationale du Canada : Canadiana
}

\section{Louis J.S. Forget}

Volume 20, numéro 4, décembre 1974

URI : https://id.erudit.org/iderudit/1055658ar

DOI : https://doi.org/10.7202/1055658ar

Aller au sommaire du numéro

\section{Éditeur(s)}

Association pour l'avancement des sciences et des techniques de la documentation (ASTED)

\section{ISSN}

0315-2340 (imprimé)

2291-8949 (numérique)

Découvrir la revue

Citer cet article

Forget, L. J. (1974). L'automatisation de la Bibliographie nationale du Canada : Canadiana. Documentation et bibliothèques, 20(4), 184-189.

https://doi.org/10.7202/1055658ar
Résumé de l'article

Cet article nous informe de l'état des travaux d'automatisation de la bibliographie nationale du Canada dans le contexte du développement d'un Système unifié d'information à la Bibliothèque nationale du Canada. On y décrit de façon générale la planification, le développement et le fonctionnement du système automatisé pour la production de Canadiana. L'objectif du système est la création d'une base de données (data base) constituée de notices bibliographiques principales comprenant tous les éléments requis pour assurer la préparation de produits bibliographiques répondant aux besoins de la Bibliothèque nationale et de sa clientèle.
Tous droits réservés (C) Association pour l'avancement des sciences et des techniques de la documentation (ASTED), 1974
Ce document est protégé par la loi sur le droit d'auteur. L'utilisation des services d'Érudit (y compris la reproduction) est assujettie à sa politique d'utilisation que vous pouvez consulter en ligne.

https://apropos.erudit.org/fr/usagers/politique-dutilisation/ 


\title{
L'automatisation de la Bibliographie nationale du Canada: Canadiana*
}

\author{
Louis J.S. Forget \\ Directeur adjoint, Systèmes, \\ Direction de la recherche et de la planification \\ Bibliothèque nationale du Canada
}

Cet article nous informe de l'état des travaux d'automatisation de la bibliographie nationale du Canada dans le contexte du développement d'un Système unifié d'information à la Bibliothèque nationale du Canada. On y décrit de façon générale la planification, le développement et le fonctionnement du système automatisé pour la production de Canadiana. L'objectif du système est la création d'une base de données (data base) constituée de notices bibliographiques principales comprenant tous les éléments requis pour assurer la préparation de produits bibliographiques répondant aux besoins de la Bibliothèque nationale et de sa clientèle.

\section{Introduction et historique}

A la Bibliothèque nationale, l'automatisation remonte au début de 1967 lorsqu'on y a entrepris une étude préliminaire pour automatiser la production de la bibliographie nationale, Canadiana. Le rapport de cette étude proposait un système d'entrée des notices bibliographiques de Canadiana sur ruban de papier perforé qui servirait ensuite au traitement par ordinateur de ces notices afin d'en produire le texte et l'index avec les renvois nécessaires. Pour plusieurs raisons, mais surtout parce que ce système était trop limité pour satisfaire aux exigences de la production de la bibliographie nationale, ce système fut rejeté. Cependant, selon un système plus flexible, on entreprit I'automatisation de l'index de Canadiana comme projet pilote. Le premier index automatisé parut dans la livraison d'avril 1968 de Canadiana.

Au même moment, aux Etats-Unis, la Library of Congress et, en Angleterre, la British National Bibliography travaillaient à la conception du format MARC. L'idée d'un format MARC est apparue avec l'automatisation dans les bibliothèques et plus particulièrement avec I'utilisation des ordinateurs électroniques pour le catalogage. Pour que l'ordinateur puisse traiter des notices bibliographiques, il faut que les divers éléments de ces notices (i.e. auteur, titre, etc.) soient identifiés ou codés et structurés de telle façon que, à l'aide de programmes d'ordinateur, on puisse-obtenir les produits et

*N.D.L.R

Nous regrettons de devoir présenter ce texte en deux parties. La livraison de mars 1975 de Documentation et bibliothèques reproduira les deux derniers chapitres de l'article.
I'information désirés de ces notices. Le format MARC peut donc être défini comme une structure à l'intérieur de laquelle on code les divers éléments d'information d'une notice bibliographique pour son traitement dans un système automatisé. II y a donc deux aspects importants à retenir dans le format MARC: la structure et le contenu.

Après l'automatisation de l'index de Canadiana, la prochaine étape devait être l'automatisation du texte complet de Canadiana. Mais cette étape a été différée afin d'entreprendre à la fin de 1968 une étude complète sur la possibilité d'automatisation de tous les services de la Bibliothèque nationale. Le rapport final de cette étude comprenant plus de 500 pages fut présenté au début de 1970: on y lisait une recommandation sur la mise en oeuvre d'un système automatisé et unifié d'information pour la Bibliothèque nationale, basé sur un système MARC dont le format serait compatible avec le format MARC II de la Library of Congress dont on prévoyait déjà qu'il deviendrait le format normalisé d'échange d'informations bibliographiques sur bandes magnétiques. Un résumé de ce rapport ${ }^{1}$ fut publié en 1970.

Pour mettre en oeuvre ce système, il fallait donc créer un format MARC qui répondrait aux besoins du Canada et qui en même temps serait compatible avec le format MARC II de la Library of Congress. Un facteur important à souligner dans I'historique du développement du format MARC canadien est le fait que la

1. Un système unifié d'information pour la Bibliothèque nationale du Canada; résumé du Rapport du Projet d'analyse et d'automatisation des services. Ottawa, Bibliothèque nationale du Canada, 1970. $88 \mathrm{p}$. 
nouvelle Loi sur la Bibliothèque nationale du Canada, entrée en vigueur le 1er septembre 1969, définissait clairement le rôle de la Bibliothèque nationale dans le domaine de l'automatisation des systèmes et réseaux de bibliothèques au pays surtout en ce qui a trait à la planification et à la normalisation.

En 1969 et 1970, plusieurs autres activités se sont déroulées concernant ce type de planification et ont servi de fondement au développement du MARC canadien. Parmi les plus importantes, soulignons le mémoire ${ }^{2}$ soumis en juin 1969 par le Directeur général de la Bibliothèque nationale au Comité spécial du Sénat de la politique scientifique. Ce mémoire comprenait 29 recommandations visant à accroìtre l'efficacité des services fournis par la Bibliothèque nationale et à mieux coordonner les services de bibliothèques sur le plan national. Durant ces années, la Bibliothèque nationale a aussi pris part aux études menées par la Télécommission établie par le ministre des Communications et, plus particulièrement, à l'étude des problèmes concernant les banques de données et les réseaux d'information.

Un fait important à noter ici est la création, au début de 1970, à la Bibliothèque nationale, d'une Direction de la recherche et de la planification chargée d'assurer la plus grande coordination possible du développement de la Bibliothèque nationale avec celui des autres bibliothèques du pays et des bibliothèques étrangères avec lesquelles la Bibliothèque nationale entretient des relations étroites et soutenues. Cette Direction joue déjà un rôle prédominant dans le perfectionnement et la coordination des services de bibliothèques du pays tout entier.

La Conférence nationale sur la normalisation du catalogage ${ }^{3}$ convoquée par le directeur général et tenue les 19 et 20 mai 1970 à la Bibliothèque nationale est une étape importante qu'il convient de souligner. Cette conférence s'imposait et ne pouvait plus être retardée. L'avènement de l'ère électronique et de ses techniques pour stocker, traiter et transmettre l'information bibliographique avait déjà eu des répercussions décisives.sur les méthodes, les techniques et les coûts de gestion des bibliothèques. A cette conférence, on a présenté neuf

2. Sénat du Canada. Délibérations du Comité spécial de la politique scientifique. Fascicule 78. Mémoire soumis par le bibliothécaire national (Appendice 188). Ottawa, juin 1969. p. 9118-9180.

3. National Conference on Cataloguing Standards, Conférence nationale sur la normalisation du catalogage. Ottawa, Bibliothèque nationale du Canada, 1970. (Conference papers, Documents de la Conférence). communications traitant surtout de l'automatisation des bibliothèques et de l'expansion des services de la Bibliothèque nationale. Ces communications ont fourni un cadre propice à la discussion au cours de laquelle on s'est attaché aux problèmes que pose la constitution d'un catalogue tant par les méthodes manuelles qu'automatiques. Les participants à cette conférence ont constaté la nécessité de coordonner les efforts d'automatisation au Canada. Afin d'en arriver à une action concrète, ils ont recommandé que la Bibliothèque nationale, en coopération avec les bibliothèques et les associations de bibliothèques canadiennes, établisse les priorités et crée des groupes de travail pour étudier des sujets tels que la normalisation du catalogage, le contenu du MARC canadien, les utilisations possibles du catalogue collectif national automatisé ou d'une banque nationale automatisée de données bibliographiques, etc.

Conformément aux recommandations de cette conférence, deux groupes de travail ont été constitués au début de 1971.

Le premier de ces groupes, le Groupe de travail sur la normalisation du catalogage, a été créé pour étudier et identifier les lacunes dans l'organisation et le traitement de la documentation canadienne et les problèmes de catalogage des bibliothèques canadiennes et, bien sûr, pour formuler des recommandations en vue d'améliorer la situation. Des normes communes de catalogage deviennent une nécessité quand il s'agit d'établir un réseau basé sur un système MARC. Ce premier groupe de travail a publié son rapport ${ }^{4}$ en 1972. On y trouve diverses recommandations sur la normalisation des vedettes, de la description bibliographique, de la classification, des vedettes-matière et du traitement des publications officielles et des publications en série.

Le deuxième de ces groupes, le Groupe de travail sur le MARC canadien, fut créé pour étudier les exigences d'un format MARC pour le Canada. C'est ce groupe de travail qui nous intéresse plus particulièrement ici.

Les membres de ce groupe de travail ont analysé et comparé les formats MARC des Etats-Unis, du Royaume-Uni, d'Italie, de France et d'Allemagne afin d'évaluer la possibilité d'adopter un de ces formats tout en satisfaisant aux exigences imposées par le bilinguisme au Canada. Ce groupe de travail a publié

4. Groupe de travail canadien sur la normalisation du catalogage. Normalisation du catalogage: rapport et recommandations. Ottawa, Bibliothèque nationale du Canada, 1973. 109 p. 
son rapport ${ }^{5}$ en 1972 et proposait un format MARC pour les monographies et un pour les publications en série. Ces formats étaient compatibles avec le MARC II américain.

Les travaux de ces deux groupes sur les questions de normalisation sont très importants pour la mise en oeuvre et le bon fonctionnement d'un réseau de bibliothèques. En effet, I'adoption d'un format MARC normalisé pour les données bibliographiques et de normes de description catalographique sont les deux principales mesures à prendre pour assurer la compatibilité des systèmes des bibliothèques canadiennes.

En 1972, la Bibliothèque nationale établissait un format MARC canadien dans le cadre de la première étape (monographies) de l'automatisation de la bibliographie nationale, Canadiana, et de son catalogage pour les livres étrangers. En 1973, elle étendait ce travail de développement aux publications en série.

En 1973, le directeur général de la Bibliothèque nationale a constitué, au sein de la Direction de la recherche et de la planification, un Bureau MARC canadien qui veille au développement et à la mise à jour du MARC canadien, et en encourage l'utilisation.

En guise de conclusion à ce bref historique, il est utile de mentionner les travaux d'un troisième groupe: celui du Groupe de travail sur le Catalogue collectif canadien, créé en 1972 , qui soumit en 1973 un rapport provisoire ${ }^{6}$, publié en 1974. Ce groupe a étudié les objectifs et le contenu du Catalogue collectif canadien ainsi que les services qui y sont reliés. II a aussi examiné le rôle du Catalogue collectif en relation avec la coopération entre bibliothèques. Ce groupe de travail a également proposé une méthodologie visant à réaliser un Catalogue collectif canadien automatisé et a établi des priorités dans la mise en application d'une banque canadienne de données bibliographiques devant servir non seulement à la localisation d'ouvrages au Canada, mais aussi au catalogage en coopération.

Ces travaux ont été suivis à la fin de 1973 et

5. MARC canadien: rapport d'activités et recommandations du Groupe de travail sur MARC concernant un format MARC canadien pour les monographies et un format MARC canadien pour les publications en série. Ottawa, Bibliothèque nationale du Canada, 1972. 253p.

6. "Groupe de travail sur le Catalogue collectif canadien: Premier rapport et recommandations intérimaires", Nouvelles de la Bibliothèque nationale, numéro spécial, janvier 1974. au début de 1974 par une étude ${ }^{7}$ plus technique sur le développement d'une base nationale canadienne de données bibliographiques incluant la mise en oeuvre d'un système de gestion de base de données et la conception d'un format Mini-MARC pour les bibliothèques participant au Catalogue collectif.

\section{Phases de l'automatisation du Sys- tème du catalogage et de Canadiana}

Les travaux d'automatisation du Système du catalogage et de Canadiana ont commencé à la fin de 1971. Ce Système du catalogage et de Canadiana est un des sous-systèmes du Système unifié d'information recommandé pour la Bibliothèque nationale dans le rapport du Projet d'analyse et d'automatisation des services. Les autres sous-systèmes couvrent les acquisitions, le contrôle des publications en série et le catalogue collectif pour les livres, les publications en série et les documents audio-visuels.

Le Système du catalogage et de Canadiana couvre toutes les opérations de catalogage pour les documents (monographies, publications en série et documents audio-visuels) que la Bibliothèque nationale achète à l'étranger pour enrichir ses collections ainsi que tous les documents reçus par le dépôt légal. II permet aussi, à partir de la base de données, de constituer divers produits tels que les fiches de catalogue, les listes, les bandes magnétiques pour le service de distribution des notices MARC ainsi que le texte et l'index de Canadiana. Le fondement du Système du catalogage et de Canadiana est donc la création d'une base de données à partir des notices bibliographiques principales lisibles par machine (MARC) comprenant toutes les données bibliographiques requises pour assurer la préparation des produits bibliographiques répondant aux besoins de la Bibliothèque nationale et de sa clientèle (voir Annexe A).

La complexité du système et la nécessité de créer des formats MARC pour chacun des différents types de publications nous ont forcés à développer le Système du catalogage et de Canadiana en trois phases (voir Annexe B).

\section{Phase I: monographies}

Le développement de cette phase qui couvre les monographies a commencé en décembre 1971 et fut complété en novembre 1973 afin

7. Duchesne, Roderick M. Etude sur une base nationale de données bibliographiques au Canada. Ottawa, Bibliothèque nationale du Canada, mars 1974. 79 p. 3 appendices. 
d'inclure les notices de la livraison de janvier 1974 de Canadiana. Ainsi, depuis janvier 1974 Canadiana est publié en deux sections séparées: la Section I qui couvre les parties I et II (monographies et thèses) est entièrement automatisée et la Section 2 qui couvre les autres parties III à VIII (publications en série; brochures; enregistrements sonores; films, films fixes et bandes magnétoscopiques; publications du gouvernement du Canada; et publications des gouvernements provinciaux du Canada) est produite selon l'ancien système manuel pour le texte et l'ancien système automatique pour l'index.

Depuis janvier 1974, le système est aussi utilisé pour le traitement des ouvrages publiés à l'extérieur du Canada et catalogués pour les collections de la Bibliothèque nationale. Avant que le système fonctionne en totalité en novembre 1973, il a été utilisé dans l'établissement des notices MARC et des fiches des catalogues de la Bibliothèque nationale comprenant toutes les publications parues dans les parties I et II de Canadiana 1973. Maintenant, le Système du catalogage et de.Canadiana produit les fiches de tous les catalogues de la Bibliothèque nationale y compris le catalogue collectif et aussi pour le service des épreuves de Canadiana, le texte et les index des parties I et II de Canadiana par photocomposition. II produit aussi des bandes MARC pour le projet pilote de distribution des bandes MARC canadiennes. La base de données comprend maintenant plus de 25,000 notices MARC.

Dans cette première phase, la Bibliothèque nationale a donc développé des formats MARC de traitement interne et de communication ${ }^{8}$ pour les monographies selon les recommandations du Groupe de travail sur le format MARC canadien et également basés sur le format MARC de la Library of Congress. Mais quelques variations se sont avérées nécessaires à cause de considérations techniques et parce que la $\mathrm{Bi}$ bliothèque nationale ne peut immédiatement mettre en oeuvre toutes les recommandations dans sa première application, la bibliographie nationale, Canadiana.

Depuis janvier 1974, la Bibliothèque nationale distribue toutes les semaines les bandes MARC canadiennes aux participants du projet pilote. En 1973, le Directeur général de la Bibliothèque nationale a invité un certain nombre de bibliothèques canadiennes à partici-

8. Format de communication du MARC canadien: monographies. Ottawa, Bureau MARC canadien, Direction de la recherche et de la planification, Bibliothèque nationale du Canada, 1973. (2ième édition, novembre 1974). per à ce projet. L'invitation se limitait aux institutions canadiennes qui recevaient déjà les bandes MARC II de la Library of Congress et aux bibliothèques qui avaient participé au Groupe de travail sur MARC. Ces limites ont été imposées afin que les participants forment un groupe assez restreint de bibliothécaires et d'analystes capable de commenter le format MARC canadien d'après leur expérience en tant qu'usagers. Ce projet se terminant en décembre 1974 sera suivi dès janvier 1975 de l'établissement du Service de distribution des bandes MARC canadiennes.

La mise en oeuvre de cette première phase du Système du catalogage et de Canadiana a présenté un microcosme des problèmes qui surgissent dans l'automatisation des services bibliographiques, y compris la conception d'un format, l'organisation des fichiers, le maintien d'une base de données, l'entrée des notices bibliographiques, la production de fiches de catalogue et de catalogues imprimés, le classement automatique et le traitement de données bilingues. Cependant, grâce à l'automatisation de Canadiana, la Bibliothèque nationale a résolu ces problèmes. Ainsi, la phase I de ce système constitue une expérience très précieuse pour tous les autres projets d'automatisation à la Bibliothèque nationale.

\section{Phase 1/: publications en série et publi- cations officielles}

Le développement de cette deuxième phase qui couvre les publications en série et les publications officielles (parties III, VII et VIII de Canadiana) ainsi que les brochures (partie IV) a commencé à l'automne 1973 et s'est terminé en octobre 1974. La phase II fonctionne depuis le 1er novembre 1974 afin d'inclure les notices de la livraison de janvier 1975 de Canadiana.

Plusieurs éléments du système et leurs interrelations ont dû être examinés dans le développement de cette phase. Comme pour les monographies dans la phase 1, on crée des notices principales MARC pour les publications en série et les publications officielles comprenant toutes les données bibliographiques requises pour assurer la préparation de produits bibliographiques répondant aux besoins de la Bibliothèque nationale et de sa clientèle. On a prêté une attention particulière aux exigences de format et de système à cause de la participation $^{9}$ de la Bibliothèque nationale au système international de données sur les publications en série (ISDS) de I'UNESCO et de I'emploi

9. "International Serials Data System (ISDS)", Accessible, vol. 1, no 5 (septembre 1973), p. 12-14. 
éventuel de la description bibliographique internationale normalisée des publications en série (ISBDS). Leur avènement a présenté un problème de synchronisation mais on a développé un format et un système qui permettent le traitement des notices ISBDS ou non-ISBDS en un seul système qui satisfait les exigences de Canadiana et de I'ISDS. La phase II a été conçue en corrélation avec le Système du catalogue collectif des publications en série à la Bibliothèque nationale qui prépare une nouvelle édition du catalogue collectif des publications en série en sciences humaines et sociales et ce, dans le cadre du projet CONSER (Conversion des publications en série) ${ }^{10}$ auquel la Bibliothèque nationale participe avec la Library of Congress et $d$ 'autres grandes bibliothèques américaines.
Au début de cette phase, on a développé un format MARC canadien de traitement interne pour les publications en série inspiré des recommandations du Groupe de travail sur le MARC canadien et du format MARC de la Library of Congress pour les publications en série. Plus tard, on a mis au point un format de communication MARC canadien pour les publications en série qui servira à la distribution des notices MARC canadiennes pour les publications en série dans le Service de distribution des bandes MARC canadiennes. Ce format MARC pour les publications en série tient compte de l'établissement prochain d'une base centrale de données pour les publications en série pour le Canada et des besoins de catalogage bilingue du pays.

On a aussi développé parallèlement à cette

Annexe A: Produits obtenus du système de catalogage et de Canadiana

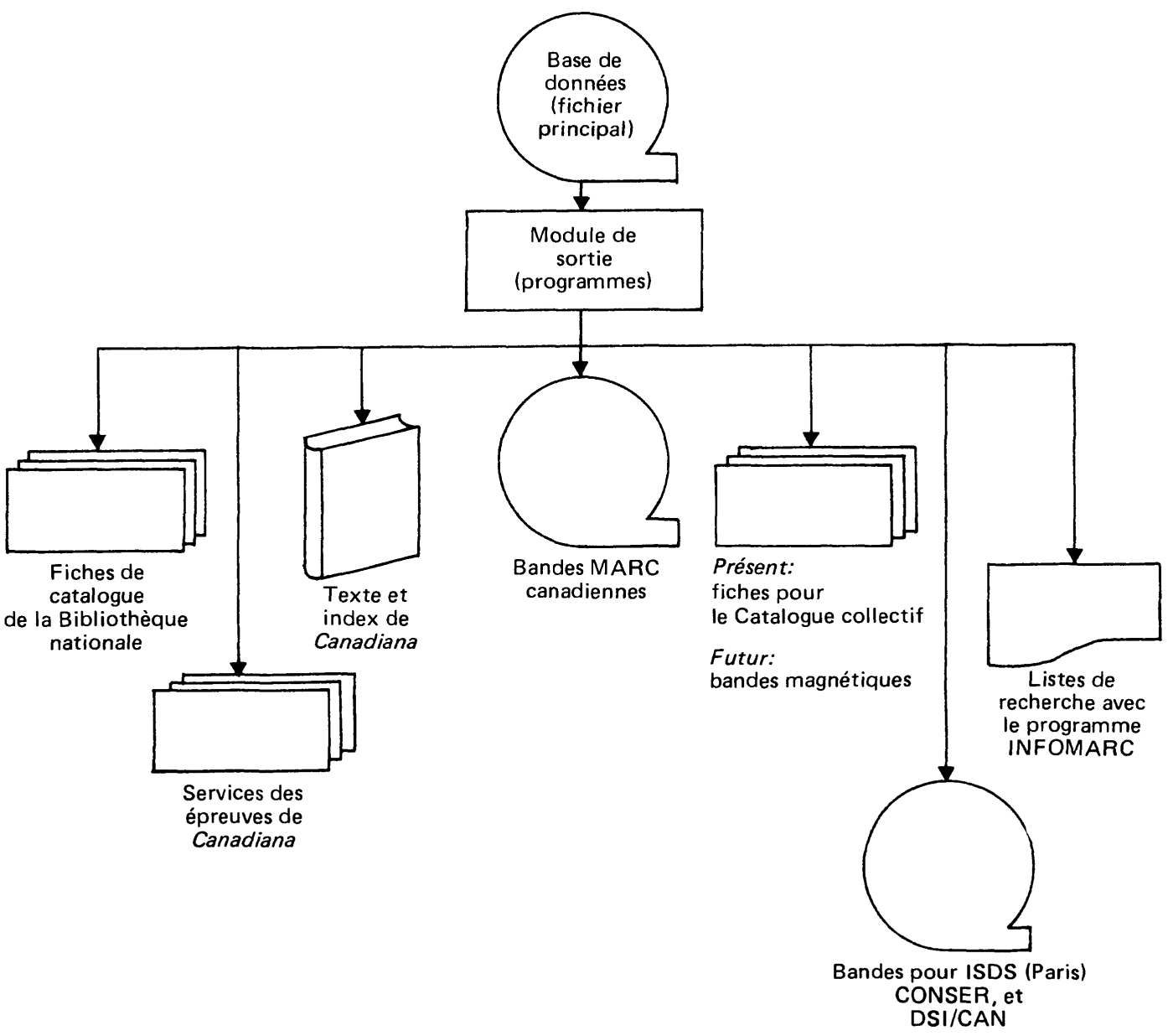

10. "Le projet CONSER (CONversion des publications en SERie", Accessible, vol. 2, no 1 (avril 1974), p. 6-7. 
phase un format MARC et un système de fichiers d'identification. 11 La conception de ce format et système qui est intégré au Système du catalogage et de Canadiana qui le sera, luimême, plus tard, au Système du Catalogue collectif a été longue et difficile à cause du peu d'expérience pratique dont nous disposons dans la conception et l'utilisation des fichiers d'identification. Ce système est presque terminé et il devrait être opérationnel à compter de janvier 1975.

\section{Phase III: documents audio-visue/s}

L'analyse et le développement de cette dernière phase commencent et on prévoit que le système fonctionnera à compter de novembre 1975 afin d'inclure les notices de la livraison de janvier 1976 de Canadiana. Cette phase couvre les parties $\mathrm{V}$ et $\mathrm{VI}$ (enregistrements sonores et films, films fixes et bandes magnétoscopiques) de Canadiana. Le bureau du MARC canadien a déjà annoncé le développement d'un format MARC canadien pour ces documents audiovisuels.

Des rapports sur le progrès des travaux de la phase III paraîtront de temps en temps dans Accessible, revue publiée et distribuée gratuitement par la Direction de la recherche et de la planification de la Bibliothèque nationale du Canada. Accessible s'adresse surtout aux bibliothèques canadiennes qui s'intéressent à l'automatisation et au développement de réseaux d'échange de données ordinolingues.

Annexe B: Étapes de l'automatisation du systèrne de catalogage et de Canadiana

\begin{tabular}{|c|c|c|c|}
\hline Description & $\begin{array}{l}\text { Date de } \\
\text { mise en } \\
\text { œuvre }\end{array}$ & Normes & $\begin{array}{c}\text { Interface } \\
\text { avec les } \\
\text { autres systèmes }\end{array}$ \\
\hline $\begin{array}{l}\text { Phase I: } \\
\text { Monographies }\end{array}$ & $\begin{array}{c}\text { Novembre } \\
1973\end{array}$ & $\begin{array}{l}\text { Règles de } \\
\text { catalogage } \\
\text { anglo-américaines } \\
\text { ISBD (M) } \\
\text { MARC canadien } \\
\text { (monographies) }\end{array}$ & $\begin{array}{l}\text { Catalogue collectif des } \\
\text { livres }\end{array}$ \\
\hline \begin{tabular}{l} 
Phase II: \\
Publications en \\
série \\
\multicolumn{1}{c}{ et } \\
publications \\
officielles
\end{tabular} & $\begin{array}{c}\text { Novembre } \\
1974\end{array}$ & $\begin{array}{l}\text { Règles de } \\
\text { catalogage } \\
\text { anglo-américaines } \\
\text { ISBD (S) } \\
\text { MARC canadien } \\
\text { (publications en } \\
\text { série) }\end{array}$ & $\begin{array}{l}\text { Catalogue collectif des } \\
\text { publications en série } \\
\text { ISDS (Paris) } \\
\text { CONSER (Conversion des } \\
\text { publications en série) }\end{array}$ \\
\hline $\begin{array}{l}\text { Phase III: } \\
\text { documents } \\
\text { audio-visuels }\end{array}$ & $\begin{array}{c}\text { Novembre } \\
1975\end{array}$ & $\begin{array}{l}\text { Règles de } \\
\text { catalogage } \\
\text { anglo-américaines } \\
\text { (révisées) } \\
\text { ISBD (AV) } \\
\text { MARC canadien } \\
\text { (documents } \\
\text { audio-visuels) }\end{array}$ & $\begin{array}{l}\text { Catalogue collectif des } \\
\text { documents audio-visuels }\end{array}$ \\
\hline
\end{tabular}

